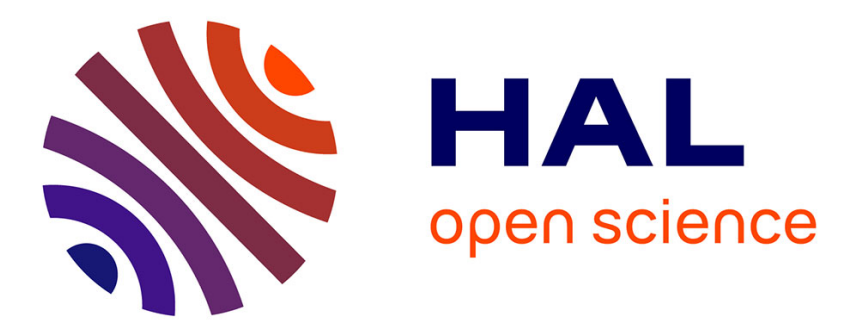

\title{
Estimation of the blood Doppler frequency shift by a time-varying parametric approach
}

Jean-Marc Girault, Denis Kouamé, Abdeldjalil Ouahabi, Frédéric Patat

\section{To cite this version:}

Jean-Marc Girault, Denis Kouamé, Abdeldjalil Ouahabi, Frédéric Patat. Estimation of the blood Doppler frequency shift by a time-varying parametric approach. Ultrasonics, 2000, 38 (1-8), pp.682 687. 10.1016/S0041-624X(99)00115-8 . hal-01076481

\section{HAL Id: hal-01076481 https://hal.science/hal-01076481}

Submitted on 22 Oct 2014

HAL is a multi-disciplinary open access archive for the deposit and dissemination of scientific research documents, whether they are published or not. The documents may come from teaching and research institutions in France or abroad, or from public or private research centers.
L'archive ouverte pluridisciplinaire HAL, est destinée au dépôt et à la diffusion de documents scientifiques de niveau recherche, publiés ou non, émanant des établissements d'enseignement et de recherche français ou étrangers, des laboratoires publics ou privés. 


\title{
Estimation of the blood Doppler frequency shift by a time- varying parametric approach
}

\author{
J.M Girault , D. Kouamé, A. Ouahabi and F. Patat. \\ GIP Ultrasons / LUSSI, 2 bis boulevard Tonnellé, 37032 Tours Cedex, France.
}

\begin{abstract}
Doppler ultrasound is widely used in medical applications to extract the blood Doppler flow velocity in the arteries via spectral analysis.

The spectral analysis of non-stationary signals and particularly Doppler signals requires adequate tools that should present both good time and frequency resolutions. It is well-known that the most commonly used time-windowed Fourier transform, which provides a time-frequency representation, is limited by the intrinsic trade-off between time and frequency resolutions. Parametric methods have then been introduced as an alternative to overcome this resolution problem. However, the performances of those methods deteriorate when high non-stationarities are present in the Doppler signal. For the purpose of accurately estimating the Doppler frequency shift, even when the temporal flow velocity is rapid (high non-stationarity), we propose to combine the use of the time-varying auto-regressive method and the (dominant) pole frequency. This proposed method performs well in the context where non-stationarities are very high. A comparative evaluation has been made between classical (FFT based) and auto-regressive (both block and recursive) algorithms. Among recursive algorithms we test an adaptive recursive method as well as a time-varying recursive method.

Finally, the superiority of the time-varying parametric approach in terms of frequencies tracking and of delay on the frequency estimate is illustrated on both simulated and in vivo Doppler signals.
\end{abstract}

Keywords: AR; Doppler frequency shift; Time-varying; Parametric method.

*Contact Author: $\quad$ Tel : +33 (0) 2-47-36-62-22

Fax : +33 (0) 2-47-36-61-20

e-mail : jmgirault@univ-tours.fr

\section{Introduction}

Doppler ultrasound provides a noninvasive assessment of the hemodynamic flow condition within blood vessels and cardiac cavities. Diagnostic information is extracted from the Doppler blood flow signal, which results from backscaterring of the ultrasound beam by moving red blood cells. Because of the random spatial distribution of red blood cells and the dynamics of the cardiovascular system, the Doppler signal is a time-varying random signal. The timefrequency distribution (TFD) of the Doppler blood flow signal is most often computed using time-windowed Fourier transform, and the resulting TFD is called the spectrogram. The spectrogram is computed on the assumption that the signal to be analyzed is stationary during a short time interval. It has the advantages of fast computation, but it has the main shortcoming of the trade-off between time and frequency resolutions. To increase the frequency resolution, a longer time interval is required. Thus, the stationary assumption may not be valid. In addition, the spectral components occurring in a large interval will be smeared in the time domain, resulting in a decreased time resolution. To partly solve this problem, autoregressive (AR) modeling has been used as an alternative technique [1], [2]. In fact, the frequency resolution can be enhanced, since model-based methods implicitly extrapolate the data outside the window under consideration. The problem is then shifted to that of the model identification; in particular, the order and the parameters of an AR process that best describes the limited duration of the Doppler signal must be estimated.

\section{Parametric modeling}

Following the principle of the timewindowed Fourier transform (spectrogram), the power spectral density (PSD) at any given time can be also obtained by applying classical autoregressive (AR) modeling.

\subsection{Nonstationary Autoregressive modeling}

The AR method consists of modeling the Doppler signal as the output of a linear filter driven by a white noise. This filter, referred to as AR, is a linear combination of the previous samples (Regressive) of the output itself (Auto). For 
examples, one can refer to [1], [2] and [3]. Equation of a classical AR complex process, in a nonstationary context, is given by:

$$
x(n)=-\sum_{i=1}^{p} a_{i}(n) x(n-i)+u(n)
$$

where $a_{i}(n)$ are AR complex parameters, $p$ is the AR order (or the number of AR parameters), $u(n)$ is a white complex noise and $n$ is the sample time.

Depending on the type of nonstationaries present in the signal, two cases can be envisaged for modeling the Doppler signal. First, when the nonstationaries of $x(n)$ are low, the AR parameters can be reached by using an algorithm applied directly to the parameters $a_{i}(n)$ of expression (1). This method is referred to as the adaptive algorithm. Secondly, when the nonstationarities are strong, we can describe the AR parameters $a(n)$ by using deterministic functions; the parameter estimation algorithm is not applied directly to the parameters but to the coefficients of the deterministic functions. This method is called the timevarying algorithm.

\subsection{Time-varying autoregressive modeling}

Now, suppose that the time-varying parameters are linear combinations of a set of deterministic basis time-varying functions $F_{g}(n)$. Time-varying AR parameters are expressed by:

$$
a_{i}(n)=\sum_{g=0}^{m} a_{i, g} F_{g}(n)
$$

where $a_{i, g}$ are AR constant complex coefficients and $m$ is the dimension of the time functions basis $F_{g}(n)$. Thus, a time-varying AR model for a nonstationary sample signal $x(n)$ is [4], [5], [6]:

$$
\mathrm{x}(\mathrm{n})=-\sum_{\mathrm{i}=1}^{\mathrm{p}} \sum_{\mathrm{g}=0}^{\mathrm{m}} \mathrm{a}_{\mathrm{i}, \mathrm{g}} \mathrm{F}_{\mathrm{g}}(\mathrm{n}-\mathrm{i}) \mathrm{x}(\mathrm{n}-\mathrm{i})+\mathrm{u}(\mathrm{n})
$$

The number of unknowns is multiplied by $(m+1)$, but this seems a small price to pay compared to the benefit of keeping the problem linear.

Several base functions [4] have been used in different fields, for example in speech signal processing and in tissue characterization [6]. Here we use one of the most common basis: the power of time functions [4]:

$$
\mathrm{F}_{\mathrm{g}}(\mathrm{n})=\frac{1}{\mathrm{~g} !}\left(\frac{\mathrm{n}}{\mathrm{N}}\right)^{\mathrm{g}}
$$

where $F_{0}(n)=1$ and $N$ is the sample number in the analyzed signal.

\subsection{Parameters estimation}

Many algorithms can be used to compute AR parameters required for the spectrum estimation. We can use either batch (block) or recursive (sequential) techniques. Here, we focus on the most popular ones: the adaptive Recursive Least-Squares (RLS) and the weighted time-varying RLS algorithms [3], [6]. The expression of both the weighted time-varying RLS algorithm and adaptive RLS algorithm are given, by rewriting equation (1), by:

$$
x(n)=\vartheta_{n-1}^{H} \hat{\theta}+u(n)
$$

where the superscript $H$ denotes the hermitian transpose operator and the hat denotes the estimate values.

The recursively estimated, the AR parameters are given by:

$$
\begin{aligned}
& \hat{\theta}_{n}=\hat{\theta}_{n-1}+P_{n} \vartheta_{n}^{*} \varepsilon_{n} \\
& \varepsilon_{n}=x_{n}-\vartheta_{n}^{H} \hat{\theta}_{n-1} \\
& P_{n}=\frac{1}{\lambda}\left(P_{n-1}-\frac{P_{n-1} \vartheta_{n}^{*} \vartheta_{n}^{H} P_{n-1}}{\lambda+\vartheta_{n}^{H} P_{n-1} \vartheta_{n}^{*}}\right)
\end{aligned}
$$

where $\hat{\theta}$ is the estimated parameters vector, $P_{n}$ the gain and $\varepsilon_{n}$ the prediction error. The superscript $*$ denotes the conjugation, $\vartheta_{n}$ is the observation vector and $\lambda$ the forgetting factor . Note that the so-called forgetting factor $\lambda$ is used to give more weight to the recent data than the past data during the estimation process. It is chosen such as $0<\lambda<1$.

In the adaptive case, the observation vector and the AR parameters become :

$$
\left\{\begin{array}{l}
\vartheta_{n}=\phi_{n}=[-x(n-1), \ldots,-x(n-p)]^{T} \\
\hat{\theta}=\left\lceil\hat{a}_{1}, \ldots, \hat{a}_{p}\right\rceil^{T}
\end{array}\right.
$$

where $T$ denotes the transpose operator.

In the time-varying case, the observation vector and the AR parameters become :

$$
\begin{aligned}
& \vartheta_{n-1}^{H}=\phi_{n-1}^{H} V \\
& V=\left[F_{0, n-1}, \ldots, F_{m, n-p}\right]^{T} \\
& \hat{\theta}=\left\lceil\hat{a}_{1,0} \ldots \hat{a}_{1, p} \ldots \hat{a}_{m, 0} \ldots \hat{a}_{m, p}\right\rceil^{T}
\end{aligned}
$$

Due to the recursive nature of this algorithm, first a transient region appears and secondly an initialization of the gain $P_{n}$ and AR parameters is required. A standard initialization value of the gain is $P_{0}=\alpha I$ (I is an identity matrix) and $\alpha \geq 10$.

In practice, the difference (called the prediction error) between the measured signal and its model is weighted by an exponential window that moves with the data: this results both in a better 
estimate of the model parameters and in the capability to track varying signals. This algorithm updates the parameters vector upon acquisition of a new sample without inverting a matrix. Note that the performances of such algorithms depend strongly upon the choice of the forgetting factor $\lambda$, which controls the length of the prediction error and therefore the amount of memory in the system [3].

Since the main purpose of our study is to extract a piece of information which resumes the temporal evolution of the Doppler frequency, we use a low AR order. This approach is slightly different from those based on a singular value decomposition (SVD) as proposed in [7] and [8]. For example, in a complex case, a first order AR model can efficiently estimate the frequency which corresponds to the pole of the Doppler signal. This is possible in the particular case of high signal-to-noise ratio as encountered in continuous Doppler.

In this way, it is possible to calculate either an instantaneous spectrum or a single pole frequency, hence to study the spectral characteristics of Doppler signals even in non-stationary conditions.

\section{Spectral estimation}

From known AR parameters, a standard approach to evaluate an instantaneous frequency consists of computing first the whole spectrum, then estimating a particular frequency (the centroid frequency or the maximum energy frequency). Accordingly, the power spectral density $S_{x x}(f, n)$ of a time-varying AR process (see eq. (1)) is given at each time $n$ by:

$$
\begin{aligned}
S_{x x}(f, n) & =\frac{\sigma_{u}^{2}(n)}{\left|1+\sum_{i=1}^{p} a_{i}(n) e^{-j 2 \pi i f}\right|^{2}} \\
S_{x x}(f, n) & =\frac{\sigma_{u}^{2}(n)}{\left|\prod_{i=1}^{p}\left(z(n)-z_{i}(n)\right)\right|_{z=e^{-2 j \pi f}}^{2}}
\end{aligned}
$$

where $f$ is the normalized frequency $-0.5 \leq f \leq 0.5, \sigma_{u}^{2}$ is the noise power at time $n$ and $z_{i}$ are poles that correspond to AR parameters.

The centroid frequency is given by:

$$
f_{c}(n)=\frac{\int_{-\infty}^{+\infty} f S_{x x}(f, n) d f}{\int_{-\infty}^{+\infty} S_{x x}(f, n) d f}
$$

As it can be seen in equation (15), an estimation of the centroid frequency implies the computation of the whole spectrum. This is not appropriated for a real time application.
An interesting alternative is to evaluate directly a single frequency (having the same variation as the centroid frequency) in which is concentrated the time-frequency contents. In this case, only one frequency has to be estimated instead of the whole spectrum. This provides an important reduction of the computing time, thus allowing real time estimation. This frequency, which corresponds to the pole argument, is given by:

$$
f_{k}(n)=\frac{f_{s}}{2 \pi} \arg \left(z_{k}(n)\right)
$$

with $\left.\arg \left(\mathrm{z}_{\mathrm{i}}(\mathrm{n})\right) \in\right]-\pi, \pi\left[\right.$ and where $f_{s}$ is the sampling frequency. In our study we only consider a low AR order, i.e. $p=1$.
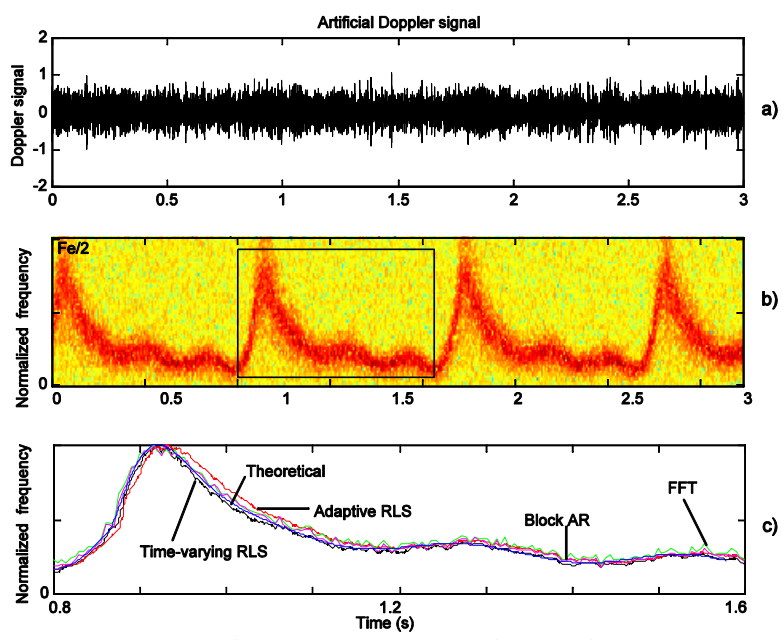

Figure 1 : Doppler frequency estimation of an artificial signal by AR methods during a 3 seconds time span. Real part of the simulated Doppler signal a). Spectrogram of the Doppler signal b). Evaluation of the Doppler frequency by combining the use of the pole frequency estimator and parametric algorithms (batch, adaptive RLS and weighted time-varying RLS), $\lambda=0.98$, and centroid frequency evaluated from the spectrogram c).

\section{Comparison}

In this part, we compare the centroid frequency computed by using the FFT to the dominant pole frequency obtained by AR methods. Three kinds of pole frequency are evaluated, each one correspond to the batch [1], the adaptive RLS [3] and the weighted time-varying RLS approaches [4], [6].

In figure (1), we present the Doppler frequency estimation, of an artificial Doppler signal, proposed by Wendling [9], evaluated by all the presented methods. These curves show that all AR algorithms give a good Doppler frequency estimation, but an advantage is in favor of the adaptive RLS and the weighted time-varying RLS approaches in terms of estimation delay and accuracy.

In order to show the typical performances of the adaptive and the time-varying methods, we 
compute an unrealistic simulated Doppler signal (see figure (2)). Though the simulated Doppler signal in figure (2) does not correspond to a true one (unrealistic), it permits to highlight some typical behaviors that cannot be easily observed in realistic simulated Doppler signals. In figure (2) a time delay between the estimated Doppler frequency (by adaptive and time-varying algorithms) and the theoretical values can be observed. This delay is more pronounced for the adaptive RLS algorithm. Note that the forgetting factor $\lambda$ for the adaptive and the weighted time-varying algorithms is the same and equal to 0.995 . This value of $\lambda$ seems a good trade-off between the estimation fluctuations and the tracking of the frequency.
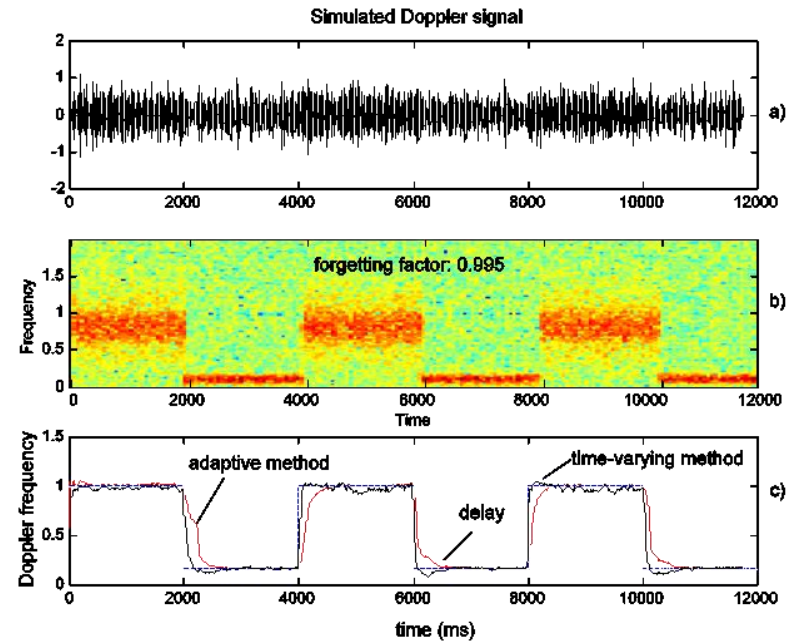

Figure 2 : Doppler frequency estimation of an unrealistic artificial signal by AR methods during a 3 seconds time span. Real part of the simulated Doppler signal a). Spectrogram of the Doppler signal b). Evaluation of the Doppler frequency by the joint use of the pole frequency estimator and AR algorithms with a forgetting factor equal to 0.995 for the two algorithms c); theoretical curve (dashed line), adaptive approach (gray solid line) and timevarying approach (dark solid line)

With in vivo Doppler signal, figure (3) shows that the Doppler frequency evaluated by the centroid estimator computed from the spectrogram has more fluctuations than the one evaluated by combining the use of the time-varying algorithm and the pole frequency estimator. This is partly due to the smoothness nature of the AR spectrum.

\section{Conclusion}

In this paper we have presented parametric methods which permit to evaluate a pole frequency. The computation time of this method is by far shorter than the one concerning the centroid frequency since it requires the evaluation of the whole spectrum. Moreover, we have compared a new parametric timevarying method to classical parametric methods. We have shown that this method provides an estimation delay lower than classical methods. This method can be used when very high nonstationarities are encountered in Doppler signals.
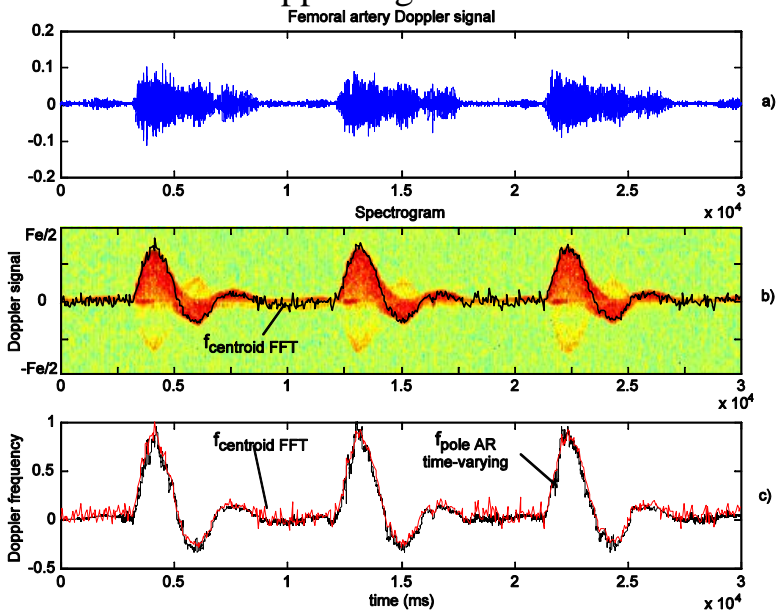

Figure 3 : Doppler frequency estimation of an in vivo femoral artery Doppler signal by the time-varying AR method; and by the joint use of the centroid frequency estimator and the spectrogram during a 3 seconds time span. Real part of the simulated Doppler signal a). Spectrogram of a real Doppler signal and its centroid frequency b). Evaluation of the Doppler frequency by the joint use of the pole frequency estimator and the time-varying AR algorithm with a forgetting factor equal to $0.98 \mathrm{c}$ ); spectrogram (gray solid line) and time-varying approach (dark solid line)

\section{References}

[1] S.M Kay and S.L. Marple, "Spectrum analysis - A modern perspective," Proc. IEEE, vol. 69, no.11, pp. 1380-1419, 1981.

[2] P.J. Vaitkus and R.S.C Cobbold, "A comparative study and assessment of Doppler ultrasound spectral estimation techniques, Part I : Estimation methods," Ultrasound in Med. \& Biol., vol. 14, no.8, pp. 661$672,1988$.

[3]L. Ljung, "Recursive Identification: Theory for the users," Englewood Cliffs, NJ: Prentice-Hall, 1987.

[4] Y. Grenier," Time-dependent ARMA modeling of nonstationary signal," IEEE Trans. Acoust., Speech, Signal Processing, vol. 31, no.4, pp. 899-911, 1983.

[5] L.A. Liporace," Linear estimation of nonstationary signal," J. Acoust. Soc. Amer., vol. 58, no.6, pp. 1288-1295, 1975.

[6] J.M Girault, F. Ossant, A. Ouahabi, D. Kouamé and F. Patat," Time-varying autoregressive Spectral estimation for ultrasound attenuation in tissue characterization," IEEE Trans. UFFC, vol. 45, no.3, pp. 650-659, 1998.

[7] F. Forsberg," On the usefulness of singular value decomposition - ARMA models in Doppler ultrasound," IEEE Trans. UFFC, vol. 38, no.5, pp. 418-428, 1991.

[8] A. Fort, C. Manfredi and S. Rocchi," Adaptive SVDBased AR model order determination for timefrequency analysis of Doppler ultrasound signals," Ultrasound in Med. \& Biol., vol. 21, no.6, pp. 793805, 1995.

[9] F. Wendling, S.A. Jones, D.P. Giddens," Simulation of Doppler ultrasound signals, pulsatile, non uniform 
Ultrasonics

flow," Ultrasound in Med. \& Biol., vol. 18, no.2, pp. 179-

193,

1992. 